\title{
Generalized procedure to determine the dependence of steady-state photoconductance lifetime on the occupation of multiple defects
}

\author{
Keith R. McIntosh, ${ }^{1, a)}$ Bijaya B. Paudyal, ${ }^{1}$ and Daniel H. Macdonald ${ }^{2}$ \\ ${ }^{1}$ Centre for Sustainable Energy Systems, Australian National University, Canberra, ACT 0200, Australia \\ ${ }^{2}$ Department of Engineering, Australian National University, Canberra, ACT 0200, Australia
}

(Received 25 July 2008; accepted 21 August 2008; published online 17 October 2008)

\begin{abstract}
We present a procedure to determine the dependence of photoconductance lifetime on the occupation of multiple defects. The procedure requires numerical iteration, making it more cumbersome than the analytical equations available for single-defect and simplified two-defect cases, but enabling the following features: (i) it accounts for the defect concentration when calculating the equilibrium carrier concentrations, (ii) it permits recombination through any number of defects, (iii) it calculates the occupation fraction of all defects at any injection, and (iv) it promotes a good understanding of the role of defect occupation in photoconductance measurements. The utility of the numerical procedure is demonstrated on an experimental sample containing multiple defects. The dependence of the sample's photoconductance on carrier concentration and temperature can be qualitatively described by the generalized procedure but not by either analytical model. The example also demonstrates that the influence of defect occupation on photoconductance lifetime measurements is mitigated at elevated temperatures-a conclusion of particular worth to the study of multicrystalline silicon. (C) 2008 American Institute of Physics. [DOI: 10.1063/1.2999640]
\end{abstract}

\section{INTRODUCTION}

Carrier lifetime $\tau_{\text {eff }}$ is routinely determined by steadystate photoconductance. ${ }^{1}$ This technique consists of measuring a sample's excess conductance $\Delta S$ as a function of photogeneration $G$, converting $\Delta S(G)$ to excess carrier concentration by assuming that the excess electron $\Delta n$ and hole $\Delta p$ concentrations are equal, and finally, by converting $\Delta n(G)$ to $\tau_{\text {eff }}(\Delta n)$ using the carrier mobility and sample width. The assumption that $\Delta n=\Delta p$ is invalid, however, when a significant fraction of light-generated carriers populates defect states rather than the conduction or valence bands. These carriers are "trapped" and do not contribute to $\Delta S$

The influence of defect occupation on $S$ was first examined in the mid-1950s by Hornbeck and Haynes ${ }^{2}$ and Fan. ${ }^{3}$ They described its best known manifestation in which there are two defects: a "recombination defect" that dominates at high carrier concentrations and a "trapping defect" that dominates at lower carrier concentrations. This two-defect trapping is observed in all multicrystalline silicon so much so that in some cases $\tau_{\text {eff }}$ cannot be measured by photoconductance at illumination levels relevant to photovoltaic operation. ${ }^{4}$ Two-defect trapping has also been observed in some monocrystalline silicon. ${ }^{5,6}$

An analytical equation that relates $S$ to the occupation of a single defect has been derived by Blakemore. ${ }^{7}$ The single defect's occupation has a subtle influence on $S$ and $\tau_{\text {eff }}$ that might easily go unnoticed. An expression for the defect concentration above which Blakemore's equation is required (i.e., when $\Delta n$ is significantly different from $\Delta p$ ) is derived by Macdonald and Cuevas ${ }^{8}$ and extended by Yashin ${ }^{9}$ to re-

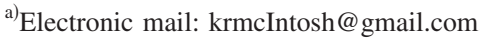

move a restriction on the permissible $\Delta n / \Delta p$ ratio. Yashin accounted for the influence of defect concentration on equilibrium concentrations. ${ }^{9}$

An analytical equation for a two-defect model has also been derived. ${ }^{2}$ It is limited, however, by two assumptions: (i) that the population and depopulation of one defect- the trapping defect-are only possible from one band (conduction or valence) and therefore, that no recombination occurs through this defect and (ii) that the defect is entirely unoccupied at equilibrium and entirely occupied under illumination. As such, it cannot account for the temperature dependence of $S$ and $\tau_{\text {eff }}$, as described in Sec. III.

In this work, we present a generalized procedure to describe the influence of defect occupation on $S$ and $\tau_{\text {eff }}$ for any number of defects. It is consistent with Blakemore's equation for a single defect and avoids the long simultaneous equations that result when extending this equation to multiple defects. It is also consistent with the Hornbeck-Haynes model when the aforementioned assumptions are valid. While more complicated than its analytical counterparts, the merits of the procedure are that (i) it accounts for the defect concentration when calculating the equilibrium carrier concentrations, (ii) it permits recombination through any number of defects, (iii) it calculates the occupation fraction of all defects at any injection, and (iv) it promotes a good understanding of the role of defect occupation in photoconductance measurements.

We first present the generalized procedure to numerically determine $S$ and $\tau_{\text {eff }}$ and then provide an example that demonstrates its utility. The example also illustrates that the debilitating effect of trapping on photoconductance lifetime measurements can be circumvented by raising the sample's 
(a) Vary $N_{t}\left(\mathrm{~cm}^{-3}\right)$

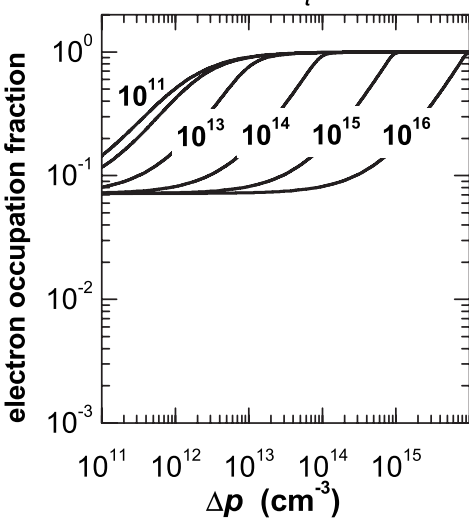

(b) Vary $\sigma_{p}\left(\mathrm{~cm}^{2}\right)$

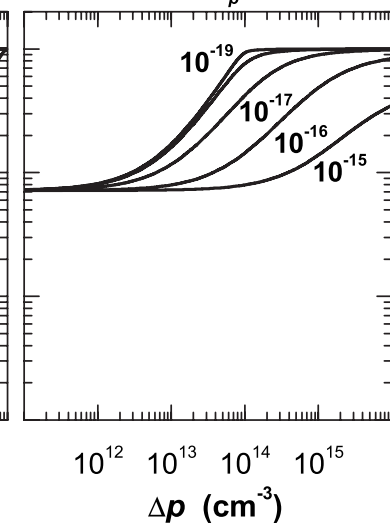

(c) Vary $E_{i}-E_{i}(\mathrm{eV})$

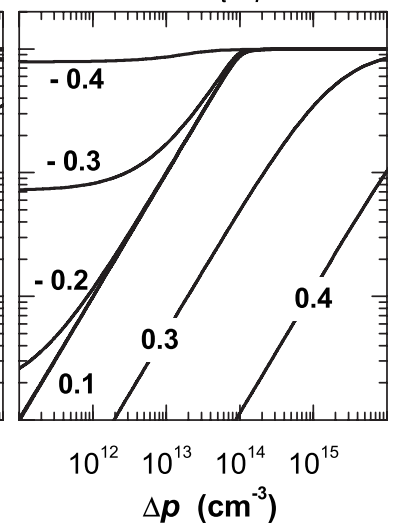

(d) $\operatorname{Vary} T(K)$

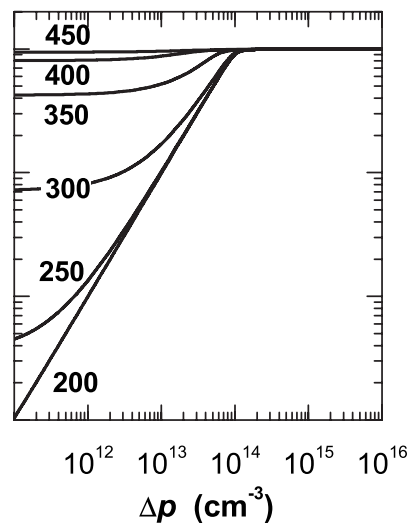

FIG. 1. Electron occupation fraction $f_{n}$ as a function of excess hole concentration $\Delta p$ and (a) trap density $N_{t}$, (b) electron capture cross section $\sigma_{n}$, (c) defect energy above the intrinsic energy $E_{t}-E_{i}$, and (d) absolute temperature $T$. Unless noted, the plots are calculated for $1 \Omega \mathrm{cm} p$-type silicon with $N_{t}$ $=10^{14} \mathrm{~cm}^{-3}, \sigma_{n}=10^{-15} \mathrm{~cm}^{2}, \sigma_{p}=10^{-19} \mathrm{~cm}^{2}, E_{t}-E_{i}=-0.3 \mathrm{eV}$, and $T=300 \mathrm{~K}$.

temperature: at an elevated temperature, the defect occupation remains constant with injection, validating the assumption that $\Delta n=\Delta p$.

\section{THEORY}

\section{A. General photoconductance lifetime theory}

A steady-state photoconductance lifetime measurement involves the comparison of a sample's conductance under photogeneration $S(G)$ to its conductance at equilibrium $S_{0}$ (i.e., $G=0$ ). For all $G, S$ depends on the free electron $n$ and hole $p$ concentrations within the sample, the mobility of those electrons $\mu_{n}$ and holes $\mu_{p}$, the width of the sample $W$, and the elementary charge $q$ by the equation

$$
S=q \int^{W}\left(\mu_{n} n+\mu_{p} p\right) d x .
$$

Being one dimensional, Eq. (1) assumes spatial uniformity in directions perpendicular to $x$.

It is usually assumed that the excess electron $\Delta n$ and hole $\Delta p$ concentrations induced by the illumination are equal,

$$
\Delta n=n-n_{0}=p-p_{0}=\Delta p,
$$

and constant with $x$ so that Eq. (1) simplifies to ${ }^{10}$

$$
\Delta S=S-S_{0}=q\left(\mu_{n}+\mu_{p}\right) \Delta n W .
$$

Equation (3) also entails the assumptions that $\Delta S$ in the surface diffusions (if present) is negligible and that $W$ is constant with $G$. [When the sample contains either a surface diffusion or surface charge, $W$ represents the width of the quasineutral bulk and can change due to depletion-region modulation (DRM). ${ }^{11-13}$ ]

Thus, with a measurement of $\Delta S(G)$ and $W$ and with a value for $\left(\mu_{n}+\mu_{p}\right)$, photoconductance measurements yield $\Delta n(G)$. One can therefore determine the sample's effective lifetime $\tau_{\text {eff }}$ in steady state $(G=U)$ as defined by ${ }^{1}$

$$
\tau_{\text {eff }}(\Delta n) \equiv \frac{\Delta n}{U(\Delta n)} .
$$

\section{B. Defect occupation fraction}

Equation (2) requires the concentration of defects occupied by electrons $n_{t}$ and holes $p_{t}$ to be the same under illumination as at equilibrium. When this is not the case, $\Delta n$ and $\Delta p$ are given by

$$
\begin{aligned}
& \Delta n=\left(n+n_{t}\right)-\left(n_{0}+n_{t 0}\right), \\
& \Delta p=\left(p+p_{t}\right)-\left(p_{0}+p_{t 0}\right),
\end{aligned}
$$

where $n_{t}=f_{n} N_{t}, p_{t}=\left(1-f_{n}\right) N_{t}, N_{t}$ is the defect concentration, and $f_{n}$ is the fraction of defects occupied by an electron, ${ }^{14}$

$$
f_{n}=\frac{\sigma_{n} n+\sigma_{p} p_{1}}{\sigma_{n}\left(n+n_{1}\right)+\sigma_{p}\left(p+p_{1}\right)},
$$

where $\sigma_{n}$ and $\sigma_{p}$ are the electron and hole capture cross sections,

$$
\begin{aligned}
& n_{1}=n_{i} \exp \left[\left(E_{t}-E_{i}\right) / k T\right], \\
& p_{1}=n_{i} \exp \left[\left(E_{i}-E_{t}\right) / k T\right],
\end{aligned}
$$

where $E_{t}$ is the energy level of the defect, $E_{i}$ is the intrinsic Fermi level, $n_{i}$ is the intrinsic carrier concentration, $k$ is the Boltzmann constant, and $T$ is the absolute temperature.

Thus, if $f_{n}$ is constant for all $G$, and therefore, for all carrier concentrations, $n_{t}$ and $p_{t}$ must also be constant (i.e., equal to $n_{t 0}$ and $p_{t 0}$ ). In this case, Eq. (5) simplifies to Eq. (2) and the theory of the previous section is valid.

There is, however, a variety of conditions for which $f_{n}$ changes significantly with carrier concentration. Figure 1 provides an example, plotting $f_{n}$ as function of the excess majority carrier concentration $\Delta p$ for $1 \Omega \mathrm{cm} p$-type silicon and as a function of (a) $N_{t}$, (b) $\sigma_{p}$, (c) $E_{t}-E_{i}$, and (d) $T$. Except for the featured parameter, the calculations of each plot were made for the baseline parameters: $N_{t}=10^{14} \mathrm{~cm}^{-3}$, $\sigma_{n}=10^{-15} \mathrm{~cm}^{2}, \sigma_{p}=10^{-19} \mathrm{~cm}^{2}, E_{t}-E_{i}=-0.3 \mathrm{eV}$, and $T$ $=300 \mathrm{~K}$. Having asymmetrical capture cross sections, a high defect concentration, and a defect energy far from the band edges, these parameters demonstrate how $f_{n}$ can vary significantly with $\Delta p$. 


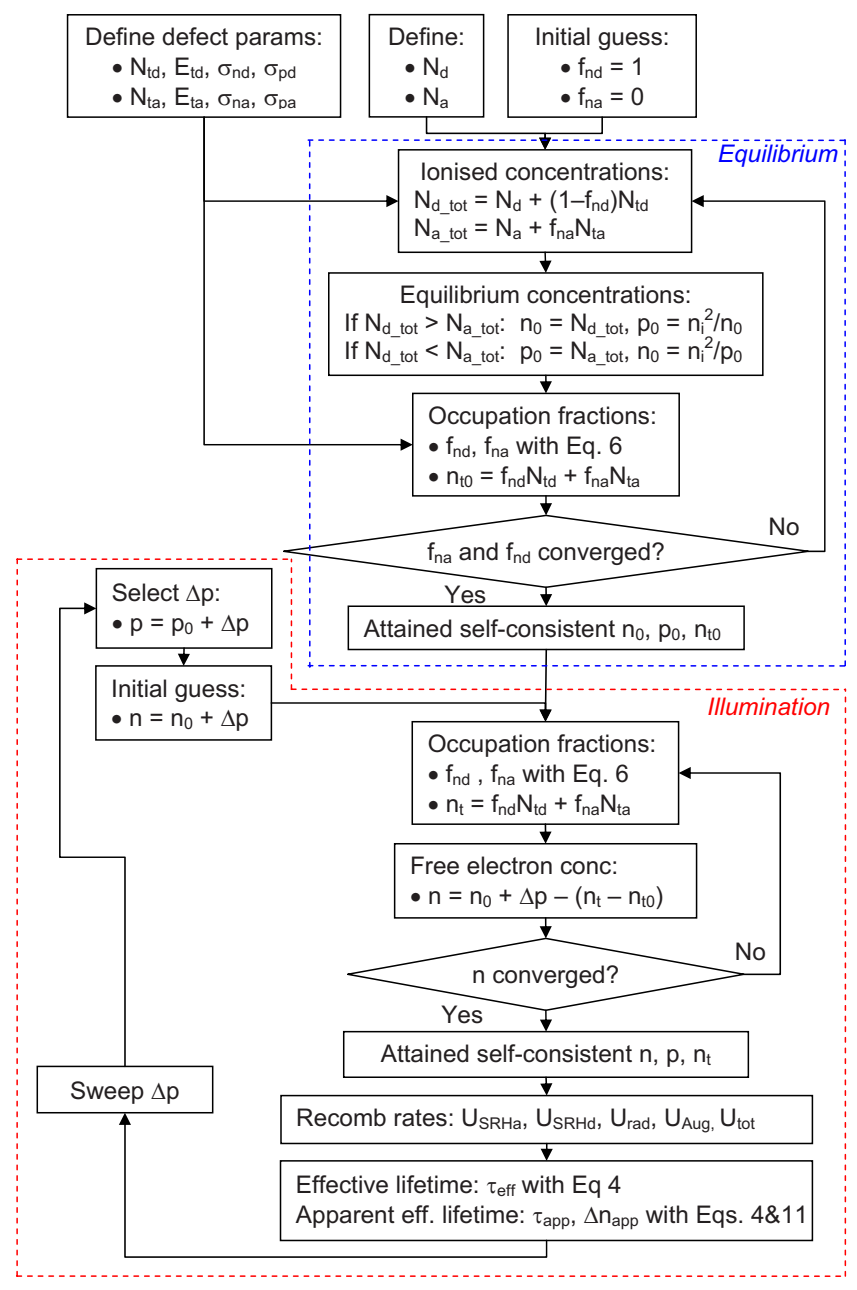

FIG. 2. (Color online) Numerical procedure to determine $f_{n}(\Delta p)$ and $\tau_{\text {eff app }}\left(\Delta n_{\text {app }}\right)$.

The most notable features of Fig. 1 are as follows. (a) The excess majority carrier concentration that fully occupies the defect increases with $N_{t}$. (b) As the ratio of minority to majority capture cross section increases, the transition from empty to occupied defects occurs more sharply at the point where the excess majority carrier concentration equals $N_{t}$. (c) The defect states are fully occupied when $E_{t}$ lies near the valence band and unoccupied when $E_{t}$ lies near the conduction band. By contrast, when $E_{t}$ is nearer midgap, the defects are mostly unoccupied when $\Delta p<N_{t}$ but fully occupied when $\Delta p>N_{t}$. (d) At low temperature, the defects are unoccupied when $\Delta p<N_{t}$ but fully occupied when $\Delta p>N_{t}$, whereas at high temperature, the defects are fully occupied at all $\Delta p$. This final plot demonstrates that $f_{n}$ at equilibrium is affected by sample temperature - a feature that is exploited in Sec. III.

\section{Procedure to account for nonconstant defect occupation}

A procedure to determine how defect occupation affects photoconductance lifetime measurements is presented in Fig. 2. This flow diagram is specific to a sample with one donorlike and one acceptorlike defect but is easily adapted to any number of defects of either type.
First, the equilibrium concentrations are determined: $n_{0}$, $p_{0}$, and $n_{t 0}$. This requires definition of the background dopant concentration for donor $N_{d}$ and acceptor $N_{a}$ atoms as well as recombination parameters associated with the donorlike defect $\left(N_{t d}, E_{t d}, \sigma_{n d}\right.$, and $\left.\sigma_{p d}\right)$ and acceptorlike defect $\left(N_{t a}, E_{t a}\right.$, $\sigma_{n a}$, and $\left.\sigma_{p a}\right)$. With an initial estimate of the defect occupation fractions $f_{n d}$ and $f_{n a}$, the total number of ionized donor $N_{d}$ tot and acceptor $N_{a}$ tot concentrations can be determined with

$$
\begin{aligned}
& N_{d \text { tot }}=N_{d}+\left(1-f_{n d}\right) N_{t d}, \\
& N_{a \text { tot }}=N_{a}+f_{n a} N_{t d},
\end{aligned}
$$

where complete ionization of all donor and acceptor atoms is assumed (although it is also possible to treat them as defects with their own energy levels instead).

If $N_{d \text { tot }}>N_{a \text { tot }}$, the sample is $n$-type and $n_{0}=N_{d}$ tot and $p_{0}=n_{i}^{2} / n_{0}$, or if $N_{d \text { tot }}<N_{a}$ tot , the sample is $p$-type and $p_{0}$ $=N_{a}$ tot and $n_{0}=n_{i}^{2} / p_{0}$. With $n_{0}$ and $p_{0}$, the new values of $f_{n d}$ and $f_{n a}$ are calculated with Eq. (6), and iteration of the above steps gives a self-consistent solution to $n_{0}, p_{0}$, and $n_{t 0}$, where

$$
n_{t 0}=f_{n d} N_{t d}+f_{n a} N_{t a} .
$$

Once the equilibrium concentrations have been attained, steady-state illumination concentrations can be determined by a slightly different approach. The simplest method is to solve for $n, p$, and $n_{t}$ at a given value of $\Delta p$ (or equivalently, $\Delta n)$. Initial values for $n$ and $p$ are chosen to be $n=n_{0}+\Delta p$ and $p=p_{0}+\Delta p . f_{n d}$ and $f_{n a}$ can then be calculated with Eq. (6) to give

$$
n=n_{0}+\Delta p-\left(n_{t}-n_{t 0}\right)
$$

and

$$
p=p_{0}+\Delta p+\left(n_{t}-n_{t 0}\right),
$$

where $n_{t}=f_{n d} N_{t d}+f_{n a} N_{t a}$. Iterating Eqs. (6) and 10 to attain convergence provides self-consistent values for $n, p$, and $n_{t}$ and therefore for $\Delta n$ and $\Delta p$ using Eq. (5).

Once the carrier concentrations are known, it is possible to calculate the Shockley-Read-Hall recombination rate through each defect, as well as Auger and radiative recombination rates. ${ }^{15}$ By summing these components (and therefore assuming no carrier hopping between defects), one attains the total recombination rate $U$ as a function of either $\Delta n$ or $\Delta p$ and therefore $\tau_{\text {eff }}$.

Finally, the photoconductance $S$ can be determined as a function of $U$ (equal to $G$ in steady state) from the free carrier concentrations and Eqs. (1) or (3). Importantly, $S(G)$ is what is determined by experimental photoconductance measurements.

\section{Apparent lifetime}

In photoconductance experiments, one rarely has knowledge of the dominating recombination parameters $\left(N_{t}, E_{t}, \sigma_{n}\right.$, and $\left.\sigma_{p}\right)$. In such a case, it is impossible to calculate $f_{n}$ and separate values for $\Delta n$ and $\Delta p$. Instead, one assumes $\Delta n$ $=\Delta p[$ Eq. (2)] to determine the apparent effective lifetime $\tau_{\text {eff app }}$ rather than the actual effective lifetime $\tau_{\text {eff. }}$ This is 

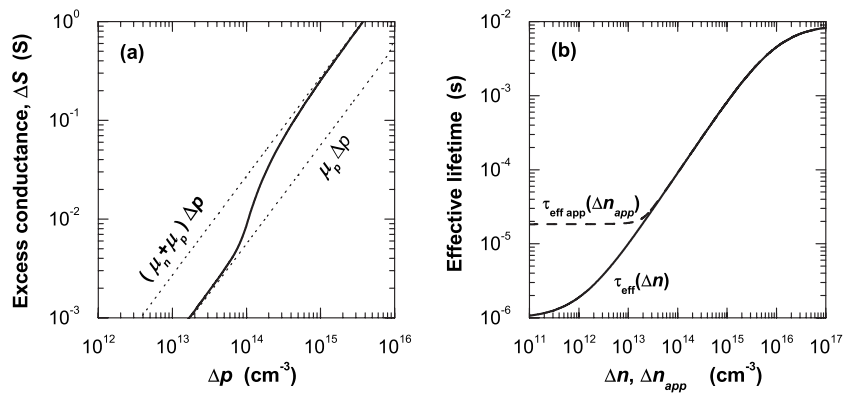

FIG. 3. (a) Excess conductance and (b) effective and apparent lifetimes calculated for the baseline conditions of Fig. 1. Note that the transition due to $f_{n}$ approaching unity occurs at $\Delta p \sim N_{t}$, or equivalently, at $\Delta n_{\text {app }}$ $\sim N_{t} \mu_{p} /\left(\mu_{n}+\mu_{p}\right)$.

achieved using the apparent excess carrier concentration $\Delta n_{\text {app }}$,

$$
\Delta n_{\mathrm{app}}=\frac{\Delta S}{q\left(\mu_{n}+\mu_{p}\right) W},
$$

and substituting $\Delta n_{\text {app }}$ into Eq. (4) to find $\tau_{\text {eff app }}$. [Almost all work published on photoconductance measurements made this assumption and therefore presented $\tau_{\text {eff app }}\left(\Delta n_{\text {app }}\right)$ rather than $\tau_{\text {eff }}(\Delta n)$.] Thus, with the application of Eqs. (11) and (4), the generalized procedure can also determine $\tau_{\text {eff }}(\Delta n)$ and $\tau_{\text {eff app }}\left(\Delta n_{\text {app }}\right)$ for any set of defect parameters.

\section{E. Deviation in apparent from actual lifetime for a single defect}

Figure 3 provides an example of how $\tau_{\text {eff app }}$ can deviate from $\tau_{\text {eff }}$ for a single defect. It plots (a) $\Delta S(\Delta p)$ and (b) $\tau_{\text {eff app }}\left(\Delta n_{\text {app }}\right)$ and $\tau_{\text {eff }}(\Delta n)$ for the baseline parameters in Fig. 1. Under sufficient illumination, $\Delta S$ is proportional to $\left(\mu_{n}\right.$ $\left.+\mu_{p}\right) \Delta p$ (Eq. (3)) because all defects are occupied and the excess carrier concentrations are much greater than the defect concentration. In this case, Eq. (2) is valid and $\tau_{\text {eff app }}$ equals $\tau_{\text {eff }}$. Under low illumination, however, $\Delta S$ is proportional to $\mu_{p} \Delta p$ because all light-generated electrons occupy the defect rather than the conduction band and do not con- tribute to the conductance. In this case, there are no additional free electrons to recombine and $U$ is constant with $\Delta p$. Thus, while one can plot $\tau_{\text {eff app }}\left(\Delta n_{\text {app }}\right)$ at low $\Delta n_{\text {app }}$, no data have actually been taken at $\Delta n$ lower than when $\tau_{\text {eff app }}$ deviates from $\tau_{\text {eff. }}$. In practice, it is difficult to know when this has occurred.

The relationship between $\tau_{\text {eff app }}\left(\Delta n_{\text {app }}\right)$ and $\tau_{\text {eff }}(\Delta n)$ can be assessed using the generalized procedure, but it can also be examined explicitly using Blakemore's model for a single defect. $^{7-9}$ Macdonald and Cuevas ${ }^{8}$ and Yashin ${ }^{9}$ derived expressions that state the conditions under which $N_{t}$ causes a deviation in $\tau_{\text {eff app }}\left(\Delta n_{\text {app }}\right)$ from $\tau_{\text {eff }}(\Delta n)$ and they described the trends between $\tau_{\text {eff app }}$ and $\tau_{\text {eff }}$ as a function of $\Delta n, N_{t}, E_{t}$, and $\sigma_{n} / \sigma_{p}$. These dependencies are complicated but can be summarized for silicon at room temperature to state that $\tau_{\text {eff app }}$ deviates from $\tau_{\text {eff }}$ when the following conditions are met: (i) the minority capture cross section is significantly larger than the majority capture cross section, (ii) $E_{t}$ is not near either band edge, and (iii) $\Delta n<N_{t}$.

It is noteworthy that the deviation in $\tau_{\text {eff app }}\left(\Delta n_{\text {app }}\right)$ from $\tau_{\text {eff }}(\Delta n)$ due to a single defect is subtle, and to the authors' knowledge, it has not been observed experimentally. With modern instrumentation, however, this might be achieved by the comparison of photoluminescence and photoconductance measurements.

\section{F. Deviation in apparent from actual lifetime for two defects}

The most easily observable experiment in which a varying $f_{n}$ can be detected is through photoconductance measurements of multicrystalline silicon. ${ }^{4,16-19}$ In some cases, $\tau_{\text {eff app }}\left(\Delta n_{\text {app }}\right)$ deviates so greatly from $\tau_{\text {eff }}(\Delta n)$ that it prevents any room-temperature assessment of recombination. Dubbed photoconductance "trapping," this artifact, which manifests as a sharp rise in $\tau_{\text {eff app }}\left(\Delta n_{\text {app }}\right)$ with decreasing $\Delta n_{\text {app }}$, cannot be explained with a single defect but requires the existence of two defects: a "recombination" defect and a trapping defect. ${ }^{2}$

Figure 4 illustrates the effect of trapping, plotting
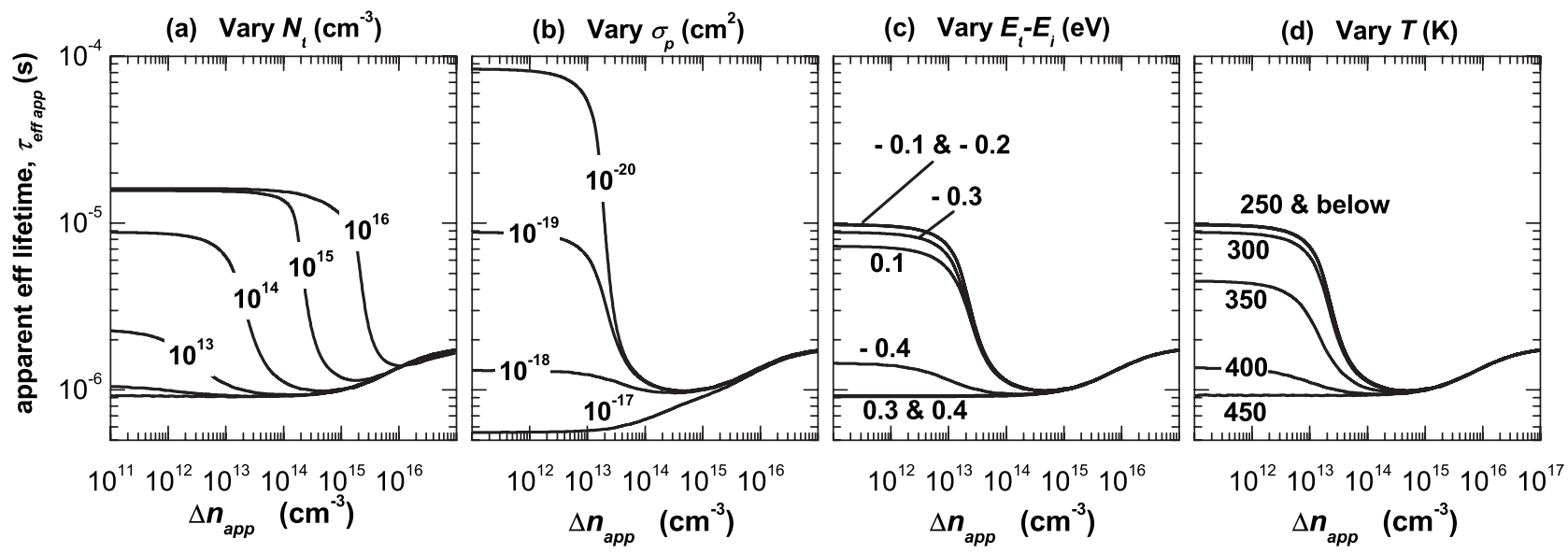

FIG. 4. Apparent effective lifetime $\tau_{\text {eff app }}$ as a function of apparent excess electron concentration $\Delta n_{\text {app }}$ for $1 \Omega \mathrm{cm} p$-type silicon with two defects at $300 \mathrm{~K}$. The first defect has $N_{t}=10^{14} \mathrm{~cm}^{-3}, \sigma_{n}=\sigma_{p}=10^{-15} \mathrm{~cm}^{2}$, and $E_{t}-E_{i}=0 \mathrm{eV}$ and the second defect has $N_{t}=10^{14} \mathrm{~cm}^{-3}, \sigma_{n}=10^{-15} \mathrm{~cm}^{2}, \sigma_{p}=10^{-19} \mathrm{~cm}^{2}$, and $E_{t}$ $-E_{i}=-0.3 \mathrm{eV}$. The figure plots the dependence of $\tau_{\text {eff app }}\left(\Delta n_{\text {app }}\right)$ on the second defect's (a) trap density $N_{t}$, (b) electron capture cross section $\sigma_{n}$, and (c) defect energy above the intrinsic energy $E_{t}-E_{i}$ as well as (d) the absolute temperature $T$. 
$\tau_{\text {eff app }}\left(\Delta n_{\text {app }}\right)$ for $1 \Omega \mathrm{cm} p$-type at $300 \mathrm{~K}$. These curves were determined by the generalized procedure for two donorlike defects, where one defect is at midgap with symmetrical capture cross sections, defined with $N_{t}=10^{14} \mathrm{~cm}^{-3}, \sigma_{n}=\sigma_{p}$ $=10^{-15} \mathrm{~cm}^{2}$, and $E_{t}-E_{i}=0 \mathrm{eV}$, and where the second trapping defect has the same parameters as those used in Fig. 1. (To permit a simple comparison between curves, the calculations omit intrinsic recombination and the temperature dependence of mobility and thermal velocity and capture cross section. It is trivial to include such effects in the generalized procedure if the dependencies are known.)

The role of the trapping defect is evident in Fig. 4. As injection decreases, there is a sudden rise in $\tau_{\text {eff app, which }}$ saturates at a value higher than the actual $\tau_{\text {eff }}$. As for a single defect, the saturation results from the recombination being limited by a lack of minority carriers, which populate defects rather than the conduction or valence bands.

Other pertinent features of Fig. 4 are as follows. (a) The

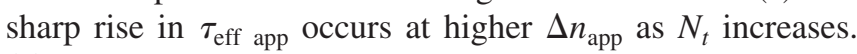
(b) The saturation level of $\tau_{\text {eff app }}$ increases as the majority carrier capture cross section of the trapping defect decreases. (c) The deviation in $\tau_{\text {eff app }}$ from $\tau_{\text {eff }}$ does not occur when the trapping defect is near either band edge because $f_{n}$ is constant. (d) The deviation in $\tau_{\text {eff app }}$ from $\tau_{\text {eff }}$ is greatest at low $T$ when $f_{n}$ of the trapping defect is zero at equilibrium, whereas $\tau_{\text {eff app }}$ does not deviate from $\tau_{\text {eff }}$ at high $T$ because $f_{n}$ is unity at all injection. These conclusions are consistent with those for Fig. 1, which plots $f_{n}$ of the trapping defect.

Prior to this work, the effect of two-defect occupation on photoconductance lifetime had been assessed with the Hornbeck-Haynes model. ${ }^{2,4,6,20,21}$ This model assumes that $f_{n}$ of the trapping defect switches from unity to zero under illumination and does not permit recombination (i.e., either $\sigma_{n}$ or $\sigma_{p}$ equals 0$)$. It has the advantage of generating an explicit equation to account for trapping on a photoconductance lifetime curve, but it does not permit a detailed assessment of all parameters as performed here. Most notably, it cannot account for changes in $f_{n}$ due to temperature-a relationship that is now exploited experimentally.

\section{EXPERIMENT}

The deviation in $\tau_{\text {eff app }}$ from $\tau_{\text {eff }}$ is sometimes considered a reason to avoid photoconductance measurements of multicrystalline silicon, ${ }^{22}$ and as evident in Fig. 4, the deviation can be vast. Figure 4(d) illustrates, however, that the deviation in $\tau_{\text {eff app }}$ from $\tau_{\text {eff }}$ is increasingly suppressed with increasing temperature. Photoconductance measurements at elevated temperature therefore provide a means to circumvent the influence of multicrystalline trapping. Temperaturecontrolled measurements also permit a more detailed characterization of the defects.

Figure 5 provides an example of temperature-controlled photoconductance lifetime measurements of a $290 \mu \mathrm{m}$ thick $1 \Omega \mathrm{cm} p$-type multicrystalline silicon wafer. A low surface recombination velocity was attained by coating the surfaces with amorphous silicon nitride by plasma-enhanced chemical vapor deposition (PECVD), ${ }^{15}$ thereby preventing surface recombination from affecting the measurements.

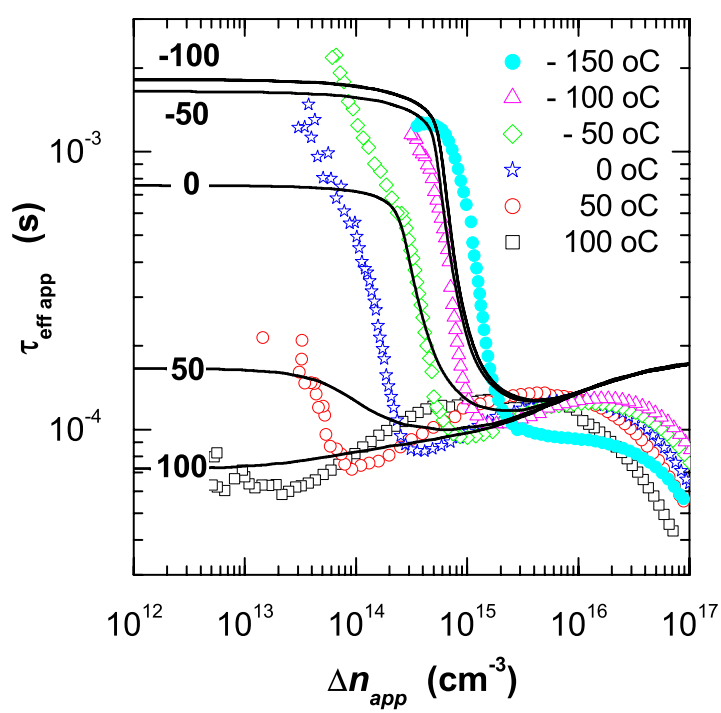

FIG. 5. (Color online) $\tau_{\text {eff app }}\left(\Delta n_{\text {app }}\right)$ for $1 \Omega \mathrm{cm} p$-type multicrystalline silicon wafer for a range of sample temperatures. The symbols represent experimental points and for clarity, only every fourth point is plotted. The lines were calculated with the generalized procedure for two defects: the first defined by $N_{t}=10^{13} \mathrm{~cm}^{-3}, \sigma_{n}=\sigma_{p}=10^{-16} \mathrm{~cm}^{2}$, and $E_{t}-E_{i}=0 \mathrm{eV}$ and the second by $N_{t}=3 \times 10^{15} \mathrm{~cm}^{-3}, \sigma_{n}=10^{-17} \mathrm{~cm}^{2}, \sigma_{p}=10^{-21} \mathrm{~cm}^{2}$, and $E_{t}$ $-E_{i}=-0.4 \mathrm{eV}$.

The experimental data (symbols) were taken using an apparatus described in Ref. 23. It exhibits the aforementioned trend of a sudden increase in $\tau_{\text {eff app }}$ with decreasing $\Delta n_{\text {app }}$ at some onset concentration $\Delta n_{\text {onset }}$ as well as a decrease in $\Delta n_{\text {onset }}$ with increasing $T$. In this example, $\Delta n_{\text {onset }}$ is less than $10^{13} \mathrm{~cm}^{-3}$ at $100{ }^{\circ} \mathrm{C}$, which is sufficiently low to analyze $\tau_{\text {eff }}$ at almost all meaningful injection levels. It is interesting that $\Delta n_{\text {onset }}$ does not saturate with decreasing temperature, indicating that the trapping defects are not entirely populated-even at the low temperature of $-150{ }^{\circ} \mathrm{C}$. At high $\Delta n_{\text {app }}, \tau_{\text {eff app }}$ decreases rapidly due to Auger recombination. Finally, while there is a faint suggestion that $\tau_{\text {eff app }}$ might saturate with decreasing $\Delta n_{\text {app }}$ at lower temperatures, $\tau_{\text {eff app }}$ continues to rise monotonically. This disagreement between theory and experiment is likely due to the existence of additional trapping defects.

The experimental data of Fig. 5 cannot be modeled by either Blakemore's equation for a single defect, which cannot produce a sudden rise in $\tau_{\text {eff app, }}$, or by Hornbeck and Haynes's equation for two defects, which does not reduce $\Delta n_{\text {onset }}$ with temperature. The trends in the data do, however, agree reasonably with those determined by the generalized procedure for two defects - as illustrated by the lines in Fig. 5. The curves were generated for a recombination defect at midgap with symmetric capture cross sections and defined by $N_{t}=10^{13} \mathrm{~cm}^{-3}, \sigma_{n}=\sigma_{p}=10^{-16} \mathrm{~cm}^{2}$, and $E_{t}-E_{i}=0 \mathrm{eV}$, and a trapping defect below midgap with asymmetric capture cross sections and defined by $N_{t}=3 \times 10^{15} \mathrm{~cm}^{-3}, \sigma_{n}$ $=10^{-17} \mathrm{~cm}^{2}, \sigma_{p}=10^{-21} \mathrm{~cm}^{2}$, and $E_{t}-E_{i}=-0.4 \mathrm{eV}$. The resulting theoretical trends agree reasonably with the experimental trends, but no attempt was made to optimize the fit between theory and experiment. Since the capture cross sections and even the defect level can change with temperature and since a variety of defects is expected to exist in the impurity laden grain boundaries, attaining high agreement 
between theory and experiment would require the fine tuning of an impractical number of parameters. What can be concluded from the simulation is that the multicrystalline silicon is reasonably well modeled as having a high concentration of defects $\left(\sim 2 \times 10^{15} \mathrm{~cm}^{-3}\right)$ with an energy in the lower half of the bandgap and with $\sigma_{n}$ much smaller than $\sigma_{p}$.

We note that the experimental data were not influenced by transient effects, as construed from varying the illumination decay constant and attaining identical results. We do not ascribe the increase in $\tau_{\text {eff app }}$ to DRM because the samples did not contain a $p-n$ junction $^{12,13}$ and because following the procedure in Ref. 11, the charge density in the PECVD silicon nitride (at most $5 \times 10^{12} \mathrm{~cm}^{-2}$ ) (Ref. 15) is insufficient to cause an increase in $\tau_{\text {eff app }}$ at a $\Delta n$ near or above $\Delta n_{\text {onset }}$.

\section{CONCLUSION}

This work presented a numerical procedure to determine a semiconductor's apparent and actual lifetimes accounting for the occupation of any number of defects. An experimental example that illustrated the utility of the procedure was provided. The example also showed that the detrimental influence of trapping in multicrystalline silicon can be mitigated by performing photoconductance measurements at elevated temperature.

\section{ACKNOWLEDGMENTS}

We thank Bruce Condon for his assistance with the temperature-controlled measurements and Bart Geerligs from the Energy Research Centre of the Netherlands (ECN) for supplying the experimental sample. This work was funded by an Australian Research Council Linkage Grant between the Australian National University, SierraTherm
Production Furnaces, and SunPower Corporation. D.M. is supported by an Australian Research Council fellowship.

${ }^{1}$ R. A. Sinton and A. Cuevas, Appl. Phys. Lett. 69, 2510 (1996).

${ }^{2}$ J. A. Hornbeck and J. R. Haynes, Phys. Rev. 97, 311 (1955).

${ }^{3}$ H. Y. Fan, Phys. Rev. 92, 1424 (1953).

${ }^{4}$ D. Macdonald and A. Cuevas, Appl. Phys. Lett. 74, 1710 (1999).

${ }^{5}$ D. Macdonald, M. Kerr, and A. Cuevas, Appl. Phys. Lett. 75, 1571 (1999).

${ }^{6}$ J. Schmidt, K. Bothe, and R. Hezel, Appl. Phys. Lett. 80, 4395 (2002).

${ }^{7}$ J. S. Blakemore, Semiconductor Statistics (Pergamon, Oxford, 1962), Sec. 8.4.

${ }^{8}$ D. Macdonald and A. Cuevas, Phys. Rev. B 67, 075203 (2003).

${ }^{9}$ A. N. Yashin, Semiconductors 39, 1285 (2005).

${ }^{10}$ D. E. Kane and R. M. Swanson, in Measurement of the Emitter Saturation Current by a Contactless Photoconductivity Decay Method, Proceedings of the 18th IEEE Photovoltaics Specialist Conference, Las Vegas, 1985 (IEEE, New York, 1985), p. 578.

${ }^{11}$ M. Bail, M. Schulz, and R. Brendel, Appl. Phys. Lett. 82, 757 (2003).

${ }^{12}$ P. J. Cousins, D. H. Neuhaus, and J. E. Cotter, J. Appl. Phys. 95, 1854 (2004).

${ }^{13}$ K. R. McIntosh, IEEE Trans. Electron Devices 54, 346 (2007)

${ }^{14}$ W. Shockley and W. T. Read, Phys. Rev. 87, 835 (1952).

${ }^{15}$ A. G. Aberle, Crystalline Silicon Solar Cells: Advanced Surface Passivation and Analysis (University of New South Wales, Sydney, 1999).

${ }^{16} \mathrm{~V}$. Yelundur, A. Rohatgi, J. W. Jeong, and J. I. Hanoka, IEEE Trans. Electron Devices 49, 1405 (2002).

${ }^{17}$ H. F. W. Dekkers, L. Carnel, and G. Beaucarne, Appl. Phys. Lett. 89, 013508 (2006).

${ }^{18}$ M. C. Schubert and W. Warta, Prog. Photovoltaics 15, 331 (2007).

${ }^{19}$ M. Dhamrin, C. Schmiga, K. Kamisako, and T. Saitoh, Sol. Energy Mater. Sol. Cells 90, 3179 (2006).

${ }^{20}$ M. C. Schubert, S. Riepe, S. Bermejo, and W. Warta, J. Appl. Phys. 99, 114908 (2006)

${ }^{21}$ P. Pohl, J. Schmidt, K. Bothe, and R. Brendel, Appl. Phys. Lett. 87, 142104 (2005)

${ }^{22}$ R. A. Bardos, T. Trupke, M. C. Schubert, and T. Roth, Appl. Phys. Lett. 88, 053504 (2006).

${ }^{23}$ B. B. Paudyal, K. R. McIntosh, D. H. Macdonald, B. S. Richards, and R. A. Sinton, Prog. Photovoltaics 16, 609 (2008). 